\title{
FDTD Calculation of Transient Electromagnetic Fields in the Grounding System of Wind Towers Due to Direct Lightning Strikes
}

\author{
Mohammad E.M. Rizk ${ }^{*}$, Ebrahim A. Badran, and Mansour H. Abdel-Rahman
}

Electrical Engineering Department, Faculty of Engineering, Mansoura University, Egypt

\begin{abstract}
Lightning protection system (LPS) for wind power generation has become an important public issue due to greatly increasing installations of wind turbines (WTs) worldwide. Grounding system is one of the most important components required for appropriate LPS for WTs. Although the finite-difference time-domain (FDTD) method for solving Maxwell's equations is difficult for computing earth potential rise (EPR) and transient grounding resistance (TGR), this method is used in this paper to compute EPR and TGR for different configurations of the grounding system of WTs because FDTD method can efficiently deal with the three-dimensional geometrical configurations of an investigated structure unlike electromagnetic transient programs (EMTP).
\end{abstract}

Keywords: Earth potential rise (EPR), electromagnetic transient programs (EMTP), finite-difference time-domain (FDTD), grounding system, hybrid arrangement, thin wire, transient grounding resistance (TGR), type A arrangement, type B arrangement.

\section{INTRODUCTION}

Lightning protection system (LPS) for wind power generation is becoming an important public issue since installations of wind turbines (WTs) have greatly increased worldwide and their generating capacities approached $282.430 \mathrm{GW}$ by the end of 2012 according to the global wind energy council (GWEC) statistics [1]. WTs are often struck by lightning because of their open-air locations, special shapes and very high construction heights. Besides seriously damaging blades, accidents take place in many wind farms where low-voltage and control circuit breakdowns frequently occur in many wind farms due to back-flow surges from the grounding systems of WTs [2].

Earthing (grounding) system of wind towers is one of the most important components required for appropriate LPSs in WTs and wind farms. Due to the relatively small foundations of wind towers when comparing with the foundations of conventional structures such as buildings, there are restrictions on the size of the grounding system of wind towers. Thus, many researches concentrate on how to get a proper design of the grounding system of wind towers which gives low grounding resistance. The earth potential rise on the grounding system of wind tower due to lightning strike decreases with lower grounding resistance. Thus, the probability of such back-flow surges and their consequences like surge arresters damages and low-voltage (and control) circuits breakdown decreases [2-4].

The FDTD method is a computing calculation algorithm in which Maxwell's electromagnetic equations are computationally treated as difference equations in both the

*Address correspondence to this author at the Electrical Engineering Department, Faculty of Engineering, Mansoura University, Egypt;

E-mail: eng_mohammad_2007@mans.edu.eg time and space domains. While the FDTD method was initially applied to electromagnetic field analysis around an antenna (Yee 1966, Kunz 1993), with the increased CPU power in PC machines, various investigations into high voltage engineering including lightning surge and grounding system analysis have also employed the algorithm [3-5].

In order to solve with FDTD method, the investigated space is divided to form a grid of cubes and then both electric and magnetic fields are computed using Maxwell's equations for each direction of the three Cartesian directions where the time derivatives and spatial derivatives are expressed by numerical differences. For each time step, both electric and magnetic fields are computed at all the cubic cells of the FDTD grid, and then the calculated electric and magnetic fields are updated with each time step. Using such method enables us to compute fields in the three dimensions unlike EMTP [6].

Thus, FDTD method is preferable to deal with the following problem types:

1. Propagation of surges in three-dimensional arrangements such as towers, and grounding systems.

2. Propagation of surges in imperfect conducting mediums such as soil.

Furthermore, by comparing FDTD method with other numerical methods for solving electromagnetic fields such as finite element method (FEM) and the method of moment (MoM), it is found that FDTD method is easier and more robust [7].

In this paper, the distribution of electric field, induced by direct lightning strike, is demonstrated in the form of contour plots for different IEC standard arrangements for grounding systems of WTs. Therefore, FDTD method is preferred as it is used to compute for the electric fields and magnetic fields by solving the Maxwell's equations in their differential form 
unlike other methods such as MoM which is used to compute for the currents by solving the Maxwell's equations in their integral form [7].

Furthermore, the earth potential rise (EPR) on the grounding system due to the lightning strike is investigated. Finally, the transient grounding resistance (TGR) is calculated for each grounding system arrangement.

\section{PROCEDURE ALGORITHM}

\subsection{Representation of Lightning Impulse Current}

Lightning inrush current which is used to investigate electromagnetic fields in the grounding system of WTs is modeled as double-exponential impulse wave. This impulse wave is defined as follows in equation (1) [8].

$I(t)=I_{0} \cdot\left(e^{-t / T_{1}}-e^{-t / T_{2}}\right)$

where, $I_{0}$ is constant in Amp., $T_{1}$, and $T_{2}$ are time constants in sec.

The lightning inrush current is defined by its front time (time to crest) which is $1 \mu \mathrm{sec}$, tail time (time to half-crest) which is $70 \mu \mathrm{sec}$, and crest (peak) value which is $30 \mathrm{kA}$ as in [3]. In order to represent this lightning inrush current as double-exponential impulse wave, equation parameters $I_{0}, T_{1}$, and $T_{2}$ should be calculated.

These parameters are calculated using Newton Raphson numerical method by knowing the front time, tail time, and peak value of the lightning impulse current [9]. Table $\mathbf{1}$ shows the constants and Fig. (1) shows the lightning current impulse wave.

\subsection{Formulation of FDTD Method}

Equations (2) and (3) demonstrate Maxwell equations in their differential form for no anisotropic and/or dispersive medium. $\nabla \times \boldsymbol{E}=-\mu \cdot \frac{\partial \boldsymbol{H}}{\partial t}$

$\nabla \times \boldsymbol{H}=\sigma \cdot \boldsymbol{E}+\varepsilon \cdot \frac{\partial \boldsymbol{E}}{\partial t}$

where, $\boldsymbol{E}$ is the electric field vector in volt $/ \mathrm{m}, \boldsymbol{H}$ is the magnetic field vector in $\mathrm{A} / \mathrm{m}, \mu$ is the magnetic permeability in $\mathrm{H} / \mathrm{m}, \sigma$ is the electric conductivity in $\mathrm{s} / \mathrm{m}$, and $\varepsilon$ is the electric permittivity in $\mathrm{F} / \mathrm{m}$.

Table 1. Lightning double exponential impulse current parameters.

\begin{tabular}{|c|c|c|}
\hline $\mathbf{I}_{\mathbf{0}}$ in $\mathbf{k A}$ & $\mathbf{T}_{\mathbf{1}}$ in $\boldsymbol{\mu s e c}$ & $\mathbf{T}_{\mathbf{2}}$ in $\boldsymbol{\mu s e c}$ \\
\hline \hline 30.35075159810815 & 99.32303118248766 & 0.154400836147643 \\
\hline
\end{tabular}

In the actual calculation, Maxwell's equations are arranged as a first-order central time-difference approximation called Yee's algorithm (Yee 1966) and the magnetic and electric fields are calculated step by step as shown in Fig. (2). From Fig. (2), it is shown that electric fields are calculated at time steps of $t=n \cdot \Delta t$, while magnetic fields at time steps of $t=(n+1 / 2) \cdot \Delta t$, where $(n=0,1, \ldots)$ alternately.

Dividing the investigated space by a small length (spatial step) of $\delta$ in the three directions, the space is filled with cubic cells of which the sides are $\delta$. The numbers of divisions of the investigated space for the Cartesian coordinate system are $N x, N y$, and $N z$ for $\mathrm{X}$-direction, $\mathrm{Y}$ direction, and $\mathrm{Z}$ direction respectively [3, 6, 10-13].

Electric and magnetic fields in equations (2) and (3) are three-dimensional vectors with three components in $\mathrm{X}$ direction, Y-direction, and Z-direction. Each equation of equations (2) and (3) are divided into three scalar equations for the three directions.

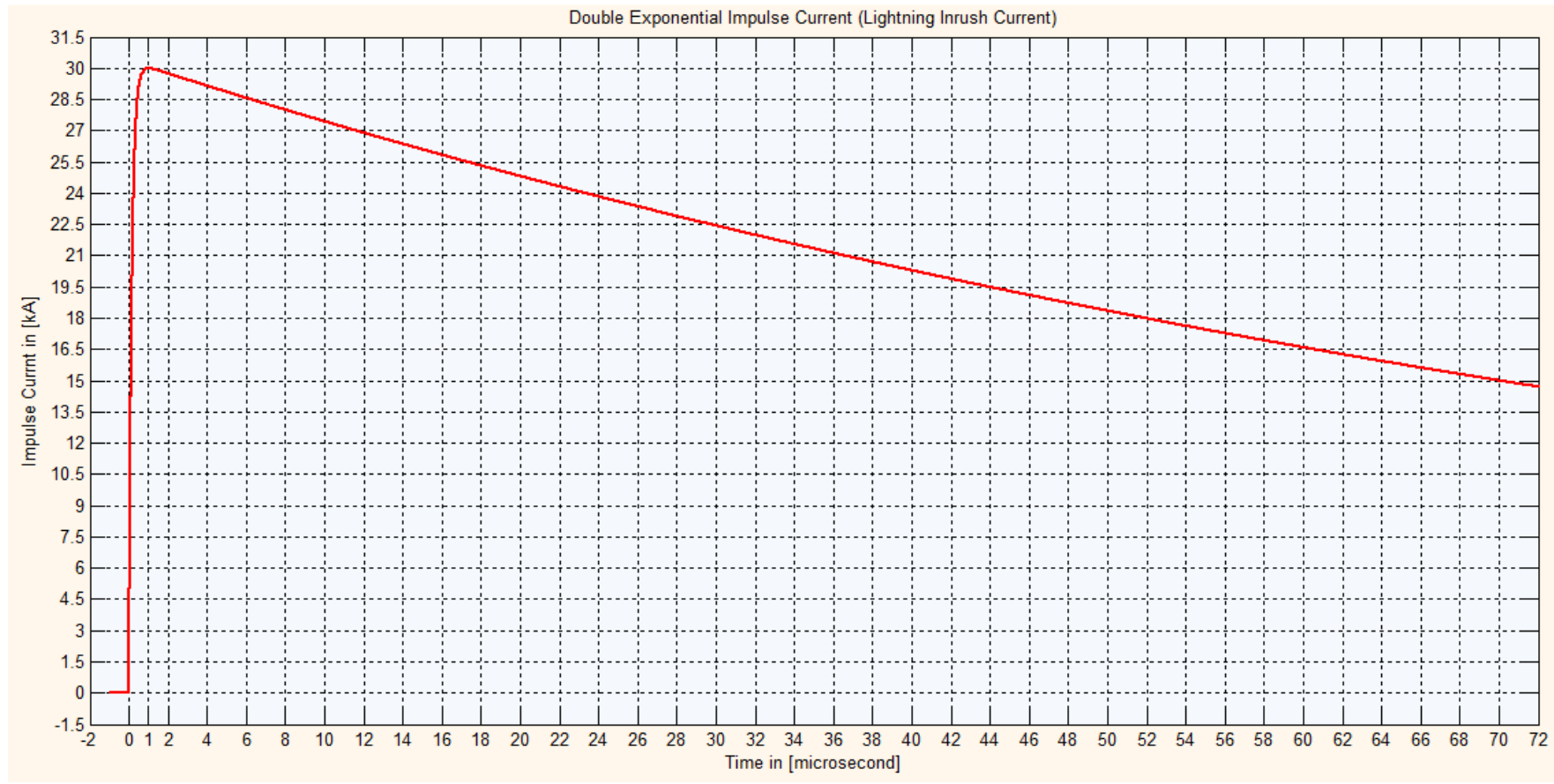

Fig. (1). Lightning double-exponential impulse current waveform. 


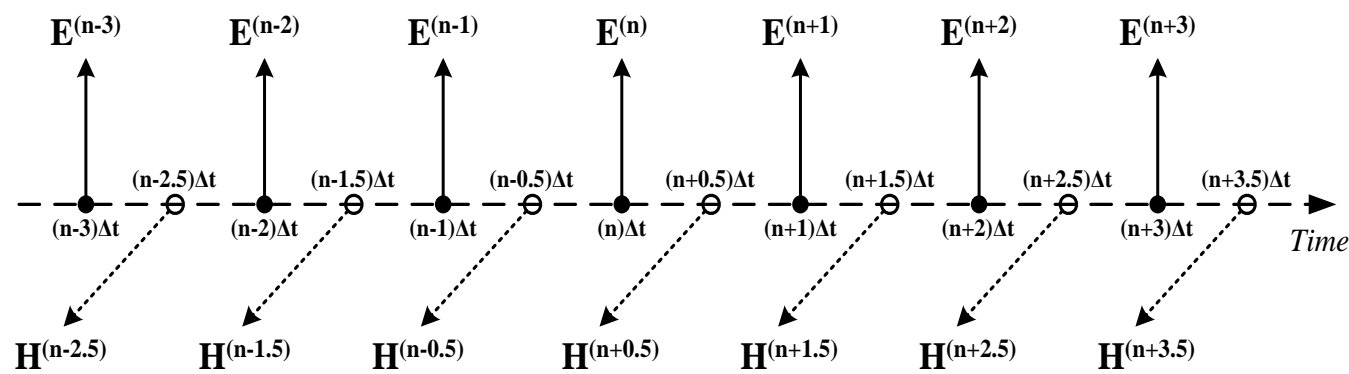

Fig. (2). Electric and magnetic fields arrangement in time-difference domain.

Thus, six scalar equations are extracted as follows in equations (4)-(9) to calculate both electric and magnetic fields in the three directions with $\mathrm{x}=\mathrm{i} \cdot \delta, \mathrm{y}=\mathrm{j} \cdot \delta$, and $\mathrm{z}=\mathrm{k}$ $\cdot \delta$, where $\mathrm{i}=1: N x, \mathrm{j}=1: N y, \mathrm{k}=1: N z$.

Fig. (3) shows a placement of electric and magnetic field components in a 3-dimension staggered mesh which is known as the Yee cell.

$$
\begin{aligned}
& \left.H_{x}{ }^{\left(n+\frac{1}{2}\right)}\left(i, j+\frac{1}{2}, k+\frac{1}{2}\right)\right) H_{\chi}^{\left(n-\frac{1}{2}\right)}\left(i, j+\frac{1}{2}, k+\frac{1}{2}\right)+\frac{\Delta t}{\mu_{\left(i, j+\frac{1}{2}, k+\frac{1}{2}\right)} \cdot \delta} . \\
& {\left[E_{y}^{(n)}{ }_{\left(i, j+\frac{1}{2}, k+1\right)}-E_{y}{ }^{(n)}\left(i, j+\frac{1}{2}, k\right)\right]-\left[E_{z}{ }^{(n)}{ }_{\left(i, j+1, k+\frac{1}{2}\right)}-\right.} \\
& \left.\left.E_{Z}^{(n)}\left(i, j, k+\frac{1}{2}\right)\right]\right] \\
& E_{x}{ }^{(n+1)}\left(i+\frac{1}{2}, j, k\right)=\frac{2 \varepsilon\left(i+\frac{1}{2}, j, k\right)-\Delta t \cdot \sigma\left(i+\frac{1}{2}, j, k\right)}{2 \varepsilon\left(i+\frac{1}{2}, j, k\right)}+E_{x}{ }_{\left(i t \cdot \frac{1}{2}, j, k\right)}^{(n)}{ }_{\left(i+\frac{1}{2^{2}}, j, k\right)}+
\end{aligned}
$$

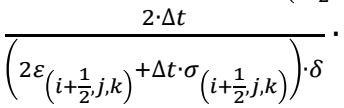

$$
\begin{aligned}
& {\left[\left[H_{Z}{ }^{\left(n+\frac{1}{2}\right)}\left(i+\frac{1}{2}, j+\frac{1}{2}, k\right)-H_{Z}^{\left(n+\frac{1}{2}\right)}\left(i+\frac{1}{2}, j-\frac{1}{2}, k\right)\right]-\right.} \\
& \left.\left[H_{y}{ }^{\left(n+\frac{1}{2}\right)}\left(i+\frac{1}{2}, j, k+\frac{1}{2}\right)-H_{y}{ }_{\left(n+\frac{1}{2}\right)}^{\left(i+\frac{1}{2}, j, k-\frac{1}{2}\right)}\right]\right]
\end{aligned}
$$

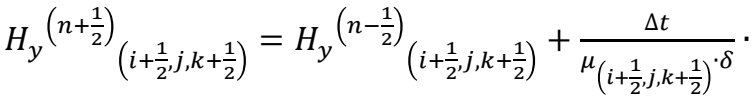

$$
\begin{aligned}
& {\left[E_{Z}^{(n)}{ }_{\left(i+1, j, k+\frac{1}{2}\right)}-E_{Z}^{(n)}\left(i, j, k+\frac{1}{2}\right)\right]-\left[E_{x}^{(n)}{ }_{\left(i+\frac{1}{2}, j, k+1\right)}-\right.} \\
& \left.\left.E_{x}^{(n)}\left(i+\frac{1}{2}, j, k\right)\right]\right]
\end{aligned}
$$

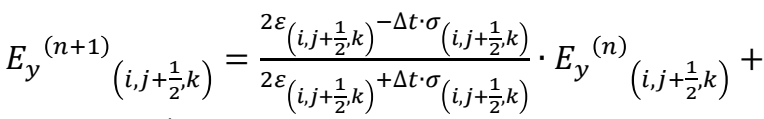

$$
\begin{aligned}
& \frac{2 \cdot \Delta t}{\left(2 \varepsilon \varepsilon_{\left(i, j+\frac{1}{2}, k\right)}+\Delta t \cdot \sigma\left(i, j+\frac{1}{2}, k\right)\right) \cdot \delta} \text {. } \\
& {\left[\left[H_{x}{ }^{\left(n+\frac{1}{2}\right)}\left(i, j+\frac{1}{2}, k+\frac{1}{2}\right)-H_{x}{ }^{\left(n+\frac{1}{2}\right)}\left(i, j+\frac{1}{2}, k-\frac{1}{2}\right)\right]-\right.} \\
& \left.\left[H_{z}{ }^{\left(n+\frac{1}{2}\right)}\left(i+\frac{1}{2}, j+\frac{1}{2}, k\right)-H_{z}{ }^{\left(n+\frac{1}{2}\right)}\left(i-\frac{1}{2}, j+\frac{1}{2}, k\right)\right]\right]
\end{aligned}
$$

$$
\begin{aligned}
& H_{Z}{ }^{\left(n+\frac{1}{2}\right)}\left(i+\frac{1}{2}, j+\frac{1}{2}, k\right)=H_{z}{ }^{\left(n-\frac{1}{2}\right)}\left(i+\frac{1}{2}, j+\frac{1}{2}, k\right)+\frac{\Delta t}{\mu_{\left(i+\frac{1}{2}, j+\frac{1}{2}, k\right)} \cdot \delta} . \\
& {\left[E_{x}^{(n)}{ }_{\left(i+\frac{1}{2}, j+1, k\right)}-E_{x}^{(n)}\left(i+\frac{1}{2}, j, k\right)\right]-\left[E_{y}^{(n)}{ }_{\left(i+1, j+\frac{1}{2}, k\right)}-\right.} \\
& \left.\left.E_{y}^{(n)}\left(i, j+\frac{1}{2}, k\right)\right]\right] \\
& E_{z}^{(n+1)}\left(i, j, k+\frac{1}{2}\right)=\frac{2 \varepsilon_{\left(i, j, k+\frac{1}{2}\right)}-\Delta t \cdot \sigma\left(i, j, k+\frac{1}{2}\right)}{2 \varepsilon_{\left(i, j, k+\frac{1}{2}\right)}+\Delta t \cdot \sigma\left(i, j, k+\frac{1}{2}\right)} \cdot E_{Z}^{(n)}\left(i, j, k+\frac{1}{2}\right)+ \\
& \left.\frac{2 \cdot \Delta t}{\left(2 \varepsilon_{\left(i, j, k+\frac{1}{2}\right)}+\Delta t \cdot \sigma\left(i, j, k+\frac{1}{2}\right)\right.}\right) \cdot \delta \\
& {\left[\left[H_{y}{ }^{\left(n+\frac{1}{2}\right)}\left(i+\frac{1}{2}, j, k+\frac{1}{2}\right)-H_{y}^{\left(n+\frac{1}{2}\right)}\left(i-\frac{1}{2}, j, k+\frac{1}{2}\right)\right]-\right.} \\
& \left.\left[H_{x}{ }^{\left(n+\frac{1}{2}\right)}\left(i, j+\frac{1}{2}, k+\frac{1}{2}\right)-H_{x}^{\left(n+\frac{1}{2}\right)}\left(i, j-\frac{1}{2}, k+\frac{1}{2}\right)\right]\right]
\end{aligned}
$$

\subsection{Accuracy and Stability}

Accurate and stable calculation of both electric and magnetic fields using FDTD method depends on the determination of spatial step, $\delta$, and time step, $\Delta \mathrm{t}$, respectively. According to the given data in section 2.1 about a front time of $1 \mu \mathrm{sec}$, lightning wave is $1 \mu \mathrm{s}$, and if the propagation velocity of the lightning impulse current wave, $v$, is equal to the light velocity of $3 \times 10^{8} \mathrm{~m} / \mathrm{s}$, so the wavelength of the lightning electromagnetic wave, $\lambda$, is 300 m [6, 10-13].

1) For Accuracy: In order to ensure accuracy of the computed results, the spatial increment, $\delta$, must be small compared to the wavelength (usually $\delta \leq \lambda / 10$ ). This amounts to have 10 or more cells per wavelength. In this paper, the spatial increment, $\delta$, is set to $0.3 \mathrm{~m}$.

2) For Stability: Moreover, in order to ensure stability of the finite difference scheme shown in the set of equations (4) - (9), the time increment, $\Delta \mathrm{t}$, must satisfy Courant's stable condition which shown in the following equation:

$\Delta t \leq \frac{1}{\sqrt{3}} \cdot \frac{\delta}{v}$ 


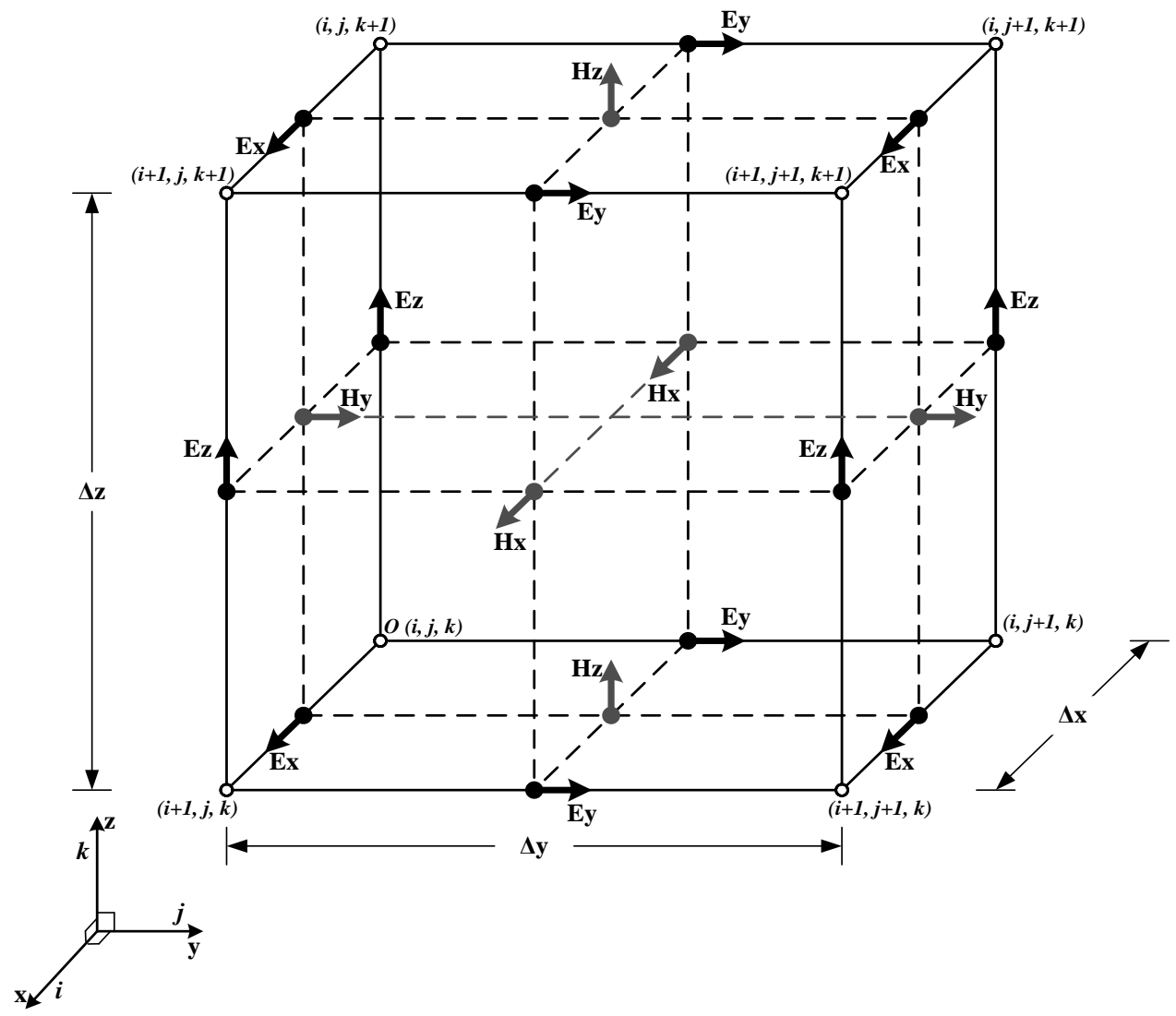

Fig. (3). Arrangement of both electric field and magnetic field in the space difference domain. (Yee cell).

Thus, in this paper, the time increment, $\Delta \mathrm{t}$, is set to $5 \times 10^{-10}$ sec to ensure that the numerical integrations of equations (4) - (9) are stable.

\subsection{Treatment of the Boundaries}

Computing the electric and magnetic fields in open (unbounded) domains represents a basic difficulty in FDTD problem because no computer can store unlimited amount of data. Therefore, an artificial boundary must be enforced to limit the extent of the solution region and to create a numerical illusion of an infinite space.

Each plane of the boundaries of the investigated space can be represented by two methods [6]. The first method assumes each plane of the boundaries as a perfectlyconducting plane so it is modeled by forcing all the tangential electric field components to be zero. The second one represents the boundaries as absorbing planes in FDTD problems using different method. In this paper, the secondorder Liao's method is used to represent the absorbing plane, because it is more widely used in such applications [6].

\subsection{Thin Wires Representation}

Thin-wires are one of the essential components in surge analysis to represent overhead lines, towers, grounding systems and so on. In order to represent such wires using FDTD method as explained before, the spatial step, $\delta$, has to be set smaller than radius of such wires. However, computers do not allow huge amount of calculations due to such a small $\delta$. The thin wire term refers to a conductor with a smaller radius than $\delta[6]$.
So far, in most FDTD analyses of transient and steadystate grounding resistance, large solid electrodes, which can be decomposed into many small cells, have been chosen, and thin-wire electrodes have not been dealt with. This is because an equivalent radius of a thin wire in a lossy medium has not been made clear, and a technique to specify an arbitrary radius of such a thin wire, which should be simple, accurate, and should not so much increase the capacity of the memory needed, has not been proposed, either.

Furthermore, an imperfectly conducting medium is required to be accurately modeled to represent currents in the earth. From the comparison between the theories of FDTD and the method of moments (MoM), the former is more advantageous to handle 3-D currents in an imperfectly conducting medium such as earth soil without any difficulty, even if the medium is non-homogeneous. On the other hand, the latter is more advantageous to accurately represent the thin wire $[10,14]$.

In this paper, a thin wire is defined as a perfectly conducting wire having a circular cross section, the radius of which is smaller than the side of the cells used. The equivalent radius of the thin wire in a lossy medium is computed by the modification of electric field and magnetic field adjacent to the thin wires as explained in [10] and [14] which develops a simple technique to specify an arbitrary radius of such a wire for more general analyses of grounding electrodes using the FDTD method. This modification in adjacent electric and magnetic field is carried out by equivalently modifying the conductivity, permittivity and 
permeability of the adjacent cells to the thin wires by a correction factor, $\mathrm{m}$, as shown in the following equations.

$r_{0} \cong 0.2298 \times \delta$

$m=\ln \left(\frac{\delta}{r_{0}}\right) / \ln \left(\frac{\delta}{r}\right)$

$\mu^{*}=\mu / m$

$\sigma^{*}=\sigma \times m$

$\varepsilon^{*}=\varepsilon \times m$

where, $r_{0}$ is the equivalent (intrinsic) thin wire radius, $r$ is the actual (arbitrary) thin wire radius, $\mu^{*}$ is the modified magnetic permeability, $\sigma^{*}$ is the modified electric conductivity, and $\varepsilon^{*}$ is the modified electric permittivity.

\subsection{Rectangular-Parallelepiped Conductors}

Electric field is always perpendicular on the conducting surfaces. Therefore, tangential components of the electric field on surfaces of the rectangular-parallelepiped conductors of the grounding systems of WTs are set be zero $[6,10]$.

\subsection{Localized Current Source}

As mentioned in section 2.1, the lightning strike is represented by a localized current source. If the current source is placed at an arbitrary point $(\mathrm{i}, \mathrm{j}, \mathrm{k})$ and the current density vector, $\mathrm{J}$, is directed along Z-direction, then it is represented by its combined magnetic field in X-direction and Y-direction as shown in equation (16) [15].

$I=\left\{\begin{array}{c}{\left[H_{x}\left(i, j+\frac{1}{2}, k\right)-H_{x}\left(i, j-\frac{1}{2}, k\right)\right]} \\ +\left[H_{y}\left(i-\frac{1}{2}, j, k\right)-H_{y}\left(i+\frac{1}{2}, j, k\right)\right]\end{array}\right\} \cdot \delta$

\section{IEC STANDARDS FOR GROUNDING SYSTEMS}

According to IEC 61400-24, there are two arrangements of earth electrodes for the grounding systems of WTs. The first one is arrangement " $\mathrm{A}$ " which comprises of horizontal or vertical electrodes connected to not less than two down conductors on the structures. The second one is arrangement "B" which comprises either of an external ring earth electrode in contact with the soil for at least $80 \%$ of its total length or a foundation earth electrode. The ring electrodes and metal parts in the foundation shall be connected to the tower structure.

Fig. (4) shows the different IEC standards for earth electrodes arrangements for grounding systems of WTs as Fig. (4a) shows arrangement "A" which comprises of 4vertical electrodes, Fig. (4b) shows arrangement "b" which comprises horizontal ring electrode, and Fig. (4c) shows hybrid arrangement which comprises of a horizontal ring electrode with 4-vertical electrodes at their 4-corners [16, 17].

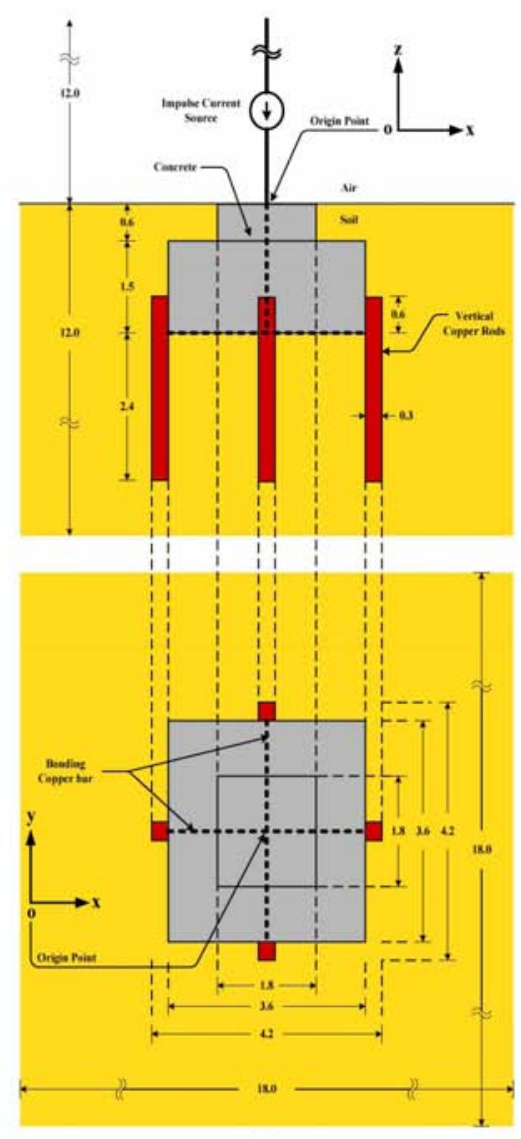

(a)

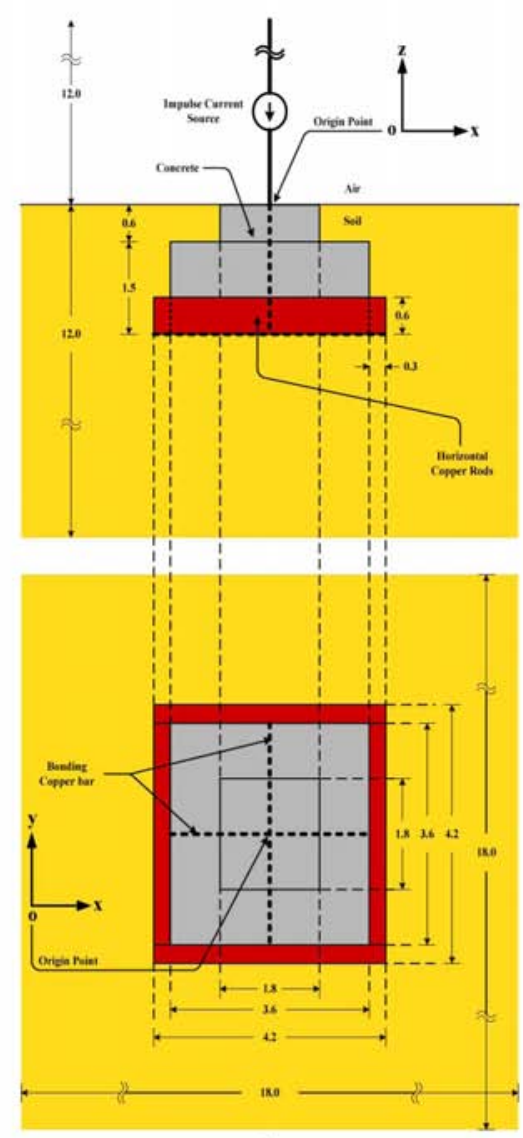

(b)

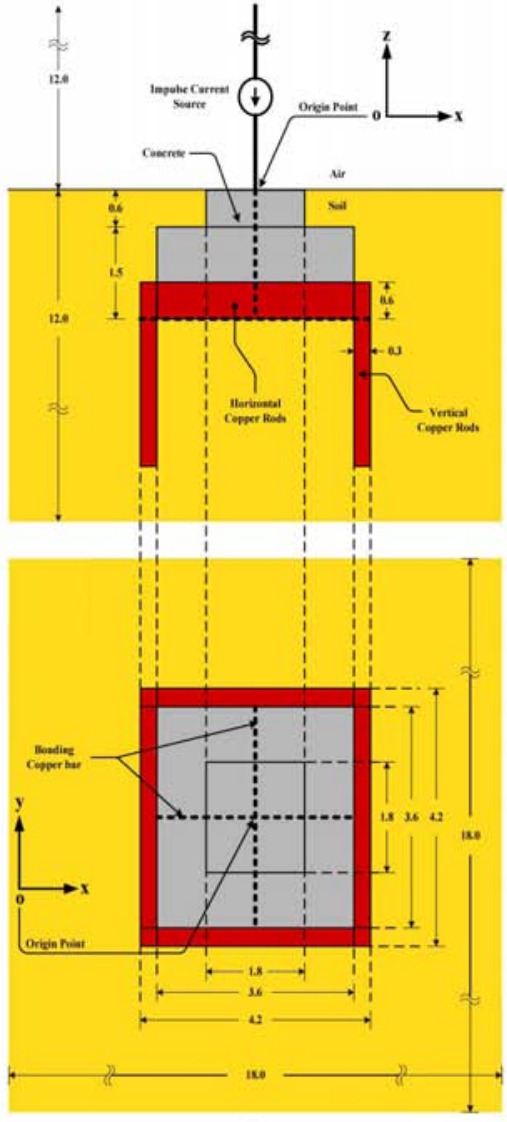

(c)

Fig. (4). IEC standard for earth electrodes for the grounding systems for WTs. (a) Arrangement "A" (4-vertical electrodes), (b) Arrangement "b" (horizontal ring electrode), (c) Hybrid arrangement (horizontal ring electrode with vertical electrodes). 


\section{FDTD CALCULATION OF ELECTROMAGNETIC FIELDS IN GROUNDING SYSTEMS}

Using FDTD method, electric and magnetic fields are calculated in each grounding system arrangement shown in Fig. (4). The space of calculation is $18 \mathrm{~m}$ in X-direction, 18 $\mathrm{m}$ in Y-direction, and $24 \mathrm{~m}$ in Z-direction as shown in Fig. (4). The electromagnetic fields calculation is carried out at three values of soil resistivity which are 2000,400 , and 80 $\Omega \cdot \mathrm{m}$. The relative electric permittivity, $\varepsilon$, and relative magnetic permeability, $\mu$, of soil are 10 and 1 respectively which are constants for all soil resistivity values. The constants of other materials in the grounding systems are shown in Table 2.
Table 2. Constants of materials.

\begin{tabular}{|c|c|c|}
\hline \multirow{2}{*}{ Relative permittivity. $\left(\varepsilon_{\mathrm{r}}\right)$} & Air & 1.0 \\
\cline { 2 - 3 } & Concrete & 6.0 \\
\hline \multirow{3}{*}{ Conductivity. $(\sigma)$} & Air & 0.0 \\
\cline { 2 - 3 } & Concrete & $58.0 \times 10^{-4}[\Omega \cdot m]^{-1}$ \\
\cline { 2 - 3 } & Copper & $58.0 \times 10^{6}[\Omega \cdot m]^{-1}$ \\
\hline Relative permeability. $\left(\mu_{\mathrm{r}}\right)$ & \multicolumn{2}{|c|}{1.0 for all materials } \\
\hline
\end{tabular}

Figs. (5-7) show the electric field distribution using contour plots at the plane, $\mathrm{Y}=0$, and at any arbitrary instant which is chosen in this paper to be $2.5 \mu$ s for all grounding

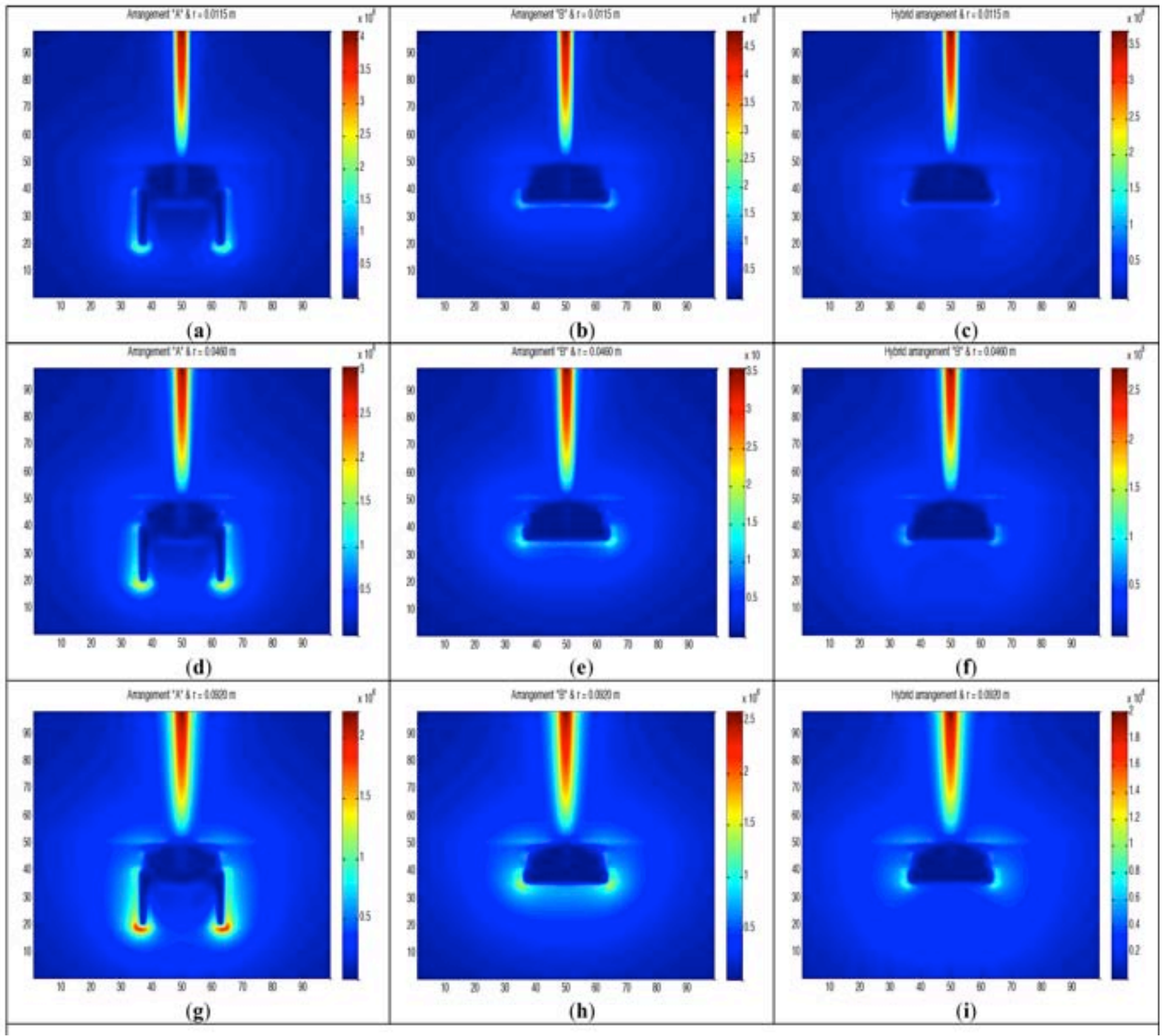

Fig. (5). Contour plot of the electric field distribution at plane $(\mathrm{Y}=0)$ in the grounding system of WT with a soil resistivity of $2000 \Omega \cdot \mathrm{m}$ at 2.5 usec. (a) at arrangement "A" and thin wire radius of $0.0115 \mathrm{~m},(\mathbf{b})$ at arrangement "B" and thin wire radius of $0.0115 \mathrm{~m},(\mathbf{c})$ at hybrid arrangement and thin wire radius of $0.0115 \mathrm{~m},(\mathbf{d})$ at arrangement " $\mathrm{A}$ " and thin wire radius of $0.0460 \mathrm{~m},(\mathbf{e})$ at arrangement "B" and thin wire radius of $0.0460 \mathrm{~m}$, (f) at hybrid arrangement and thin wire radius of $0.0460 \mathrm{~m},(\mathbf{g})$ at arrangement "A" and thin wire radius of $0.0920 \mathrm{~m},(\mathbf{h})$ at arrangement "B" and thin wire radius of $0.0920 \mathrm{~m}$, and (i) at hybrid arrangement and thin wire radius of $0.0920 \mathrm{~m}$. 


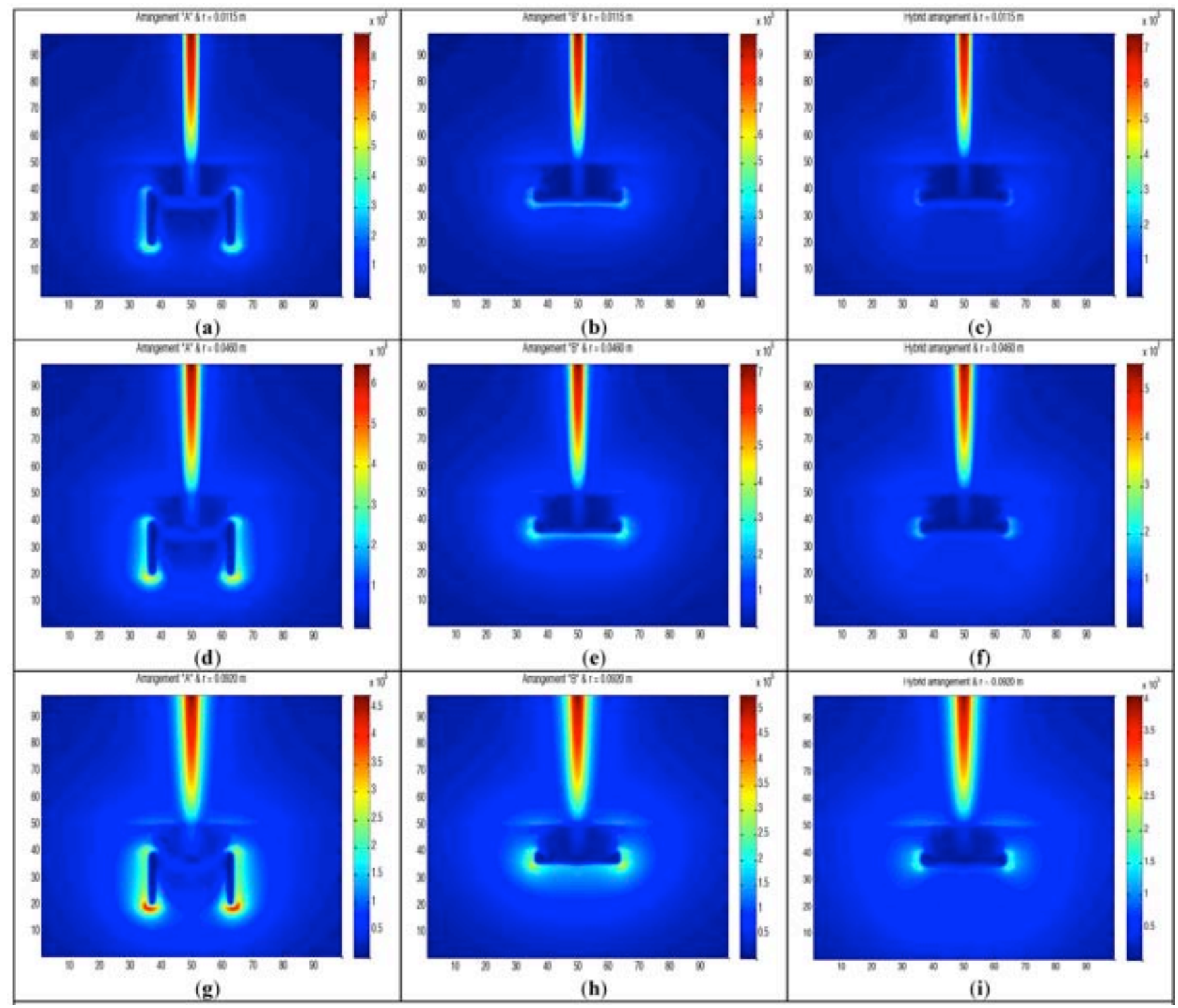

Fig. (6). Contour plot of the electric field distribution at plane (Y=0) in the grounding system of WT with a soil resistivity of $400 \Omega \cdot \mathrm{m}$ at 2.5 $\mu$ sec. (a) at arrangement " $\mathrm{A}$ " and thin wire radius of $0.0115 \mathrm{~m},(\mathbf{b})$ at arrangement "B" and thin wire radius of $0.0115 \mathrm{~m},(\mathbf{c})$ at hybrid arrangement and thin wire radius of $0.0115 \mathrm{~m},(\mathbf{d})$ at arrangement "A" and thin wire radius of $0.0460 \mathrm{~m},(\mathbf{e})$ at arrangement "B" and thin wire radius of $0.0460 \mathrm{~m}$, (f) at hybrid arrangement and thin wire radius of $0.0460 \mathrm{~m},(\mathbf{g})$ at arrangement "A" and thin wire radius of $0.0920 \mathrm{~m},(\mathbf{h})$ at arrangement "B" and thin wire radius of $0.0920 \mathrm{~m}$, and (i) at hybrid arrangement and thin wire radius of $0.0920 \mathrm{~m}$.

systems arrangements shown in Fig. (4) at soil resistivity values of $2000 \Omega \cdot \mathrm{m}, 400 \Omega \cdot \mathrm{m}$, and $80 \Omega \cdot \mathrm{m}$ respectively. These figures are arranged as $(3 \times 3)$ matrices of nine subplots for each one. Each row demonstrates the electric field distribution at a certain radius of the thin wires where (first row $\rightarrow \mathrm{r}=0.0115 \mathrm{~m}$, second row $\rightarrow \mathrm{r}=0.0460 \mathrm{~m}$, and third row $\rightarrow \mathrm{r}=0.0920 \mathrm{~m}$ ). While, each column demonstrates the electric field distribution at a certain configuration of the grounding electrodes where (first row $\rightarrow$ arrangement " $A$ ", second row $\rightarrow$ arrangement "B", and third row $\rightarrow$ hybrid arrangement).

From these figures, it is shown that the maximum value of electric field for any certain configuration decreases with the increase in the thin wire radius. However; the rate of decrease in the electric field intensity with moving away from the conducting points in the grounding system becomes less with larger radius of the thin wire. Furthermore, it is clear that for a certain thin wire radius, the maximum value of the electric field is arranged in ascending order from hybrid arrangement then arrangement " $\mathrm{A}$ " and finally arrangement "B".

\section{CALCULATION OF EARTH POTENTIAL RISE AND TRANSIENT GROUNDING RESISTANCE}

\subsection{Calculation of Earth Potential Rise}

In this section, the earth potential rise (EPR) is calculated by the integration of the electric field, which is calculated in section 4 , from the point where the lightning inrush current enters the earth along the surface of the earth to the boundary of solution using the trapezoidal numerical integration. The 


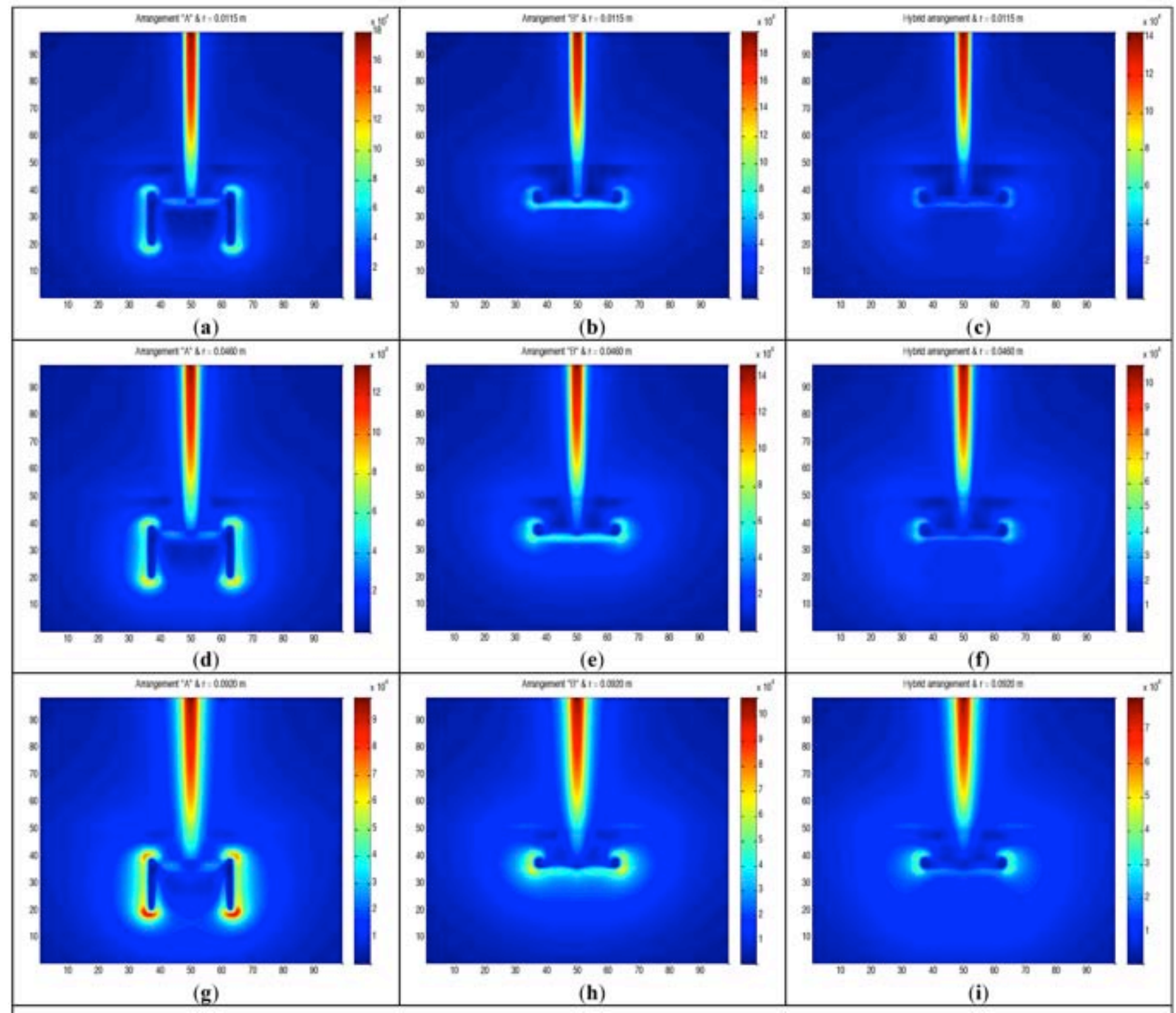

Fig. (7). Contour plot of the electric field distribution at plane $(\mathrm{Y}=0)$ in the grounding system of WT with a soil resistivity of $80 \Omega \cdot \mathrm{m}$ at 2.5 $\mu$ sec. (a) at arrangement " $\mathrm{A}$ " and thin wire radius of $0.0115 \mathrm{~m},(\mathbf{b})$ at arrangement "B" and thin wire radius of $0.0115 \mathrm{~m},(\mathbf{c})$ at hybrid arrangement and thin wire radius of $0.0115 \mathrm{~m},(\mathbf{d})$ at arrangement " $\mathrm{A}$ " and thin wire radius of $0.0460 \mathrm{~m},(\mathbf{e})$ at arrangement " $\mathrm{B}$ " and thin wire radius of $0.0460 \mathrm{~m},(\mathbf{f})$ at hybrid arrangement and thin wire radius of $0.0460 \mathrm{~m},(\mathbf{g})$ at arrangement "A" and thin wire radius of $0.0920 \mathrm{~m},(\mathbf{h})$ at arrangement "B" and thin wire radius of $0.0920 \mathrm{~m}$, and (i) at hybrid arrangement and thin wire radius of $0.0920 \mathrm{~m}$.

EPR is calculated from a time instant of zero to $2.8 \mu$ s for the three grounding arrangements which are shown in Fig. (4). For each one of these arrangements, the EPR is calculated at three values of the thin wire radius which are mentioned before in order to clarify the effect of changing the thin wire radius on EPR in FDTD calculations.

Figs. (8-10) show the EPR at three values of soil resistivity which are $2000 \Omega \cdot \mathrm{m}, 400 \Omega \cdot \mathrm{m}$, and $80 \Omega \cdot \mathrm{m}$ respectively. These figures show fluctuations in the earth surface EPR during the front period of the lightning current wave. The fluctuations are less with higher soil resistivity at the same permittivity and permeability since soil resistivity causes damping to EPR fluctuations. These fluctuations appear as overshoots in both Figs. $(\mathbf{9}, \mathbf{1 0})$ where the overshoot is more severe in Fig. (10) due to the lower value of the soil resistivity. A zoom in is done after the overshoots in both Figs. $(9,10)$ from the instant of $0.6 \mu$ s to $2.8 \mu \mathrm{s}$ in order to clarify the effect of changing the thin wire radius on the surface EPR after the period when the overshoots occur.

From Figs. (8-10), it is obvious that the effect of changing the thin wire radius increases with lower soil resistivity. During the period when the fluctuations in surface EPR occur, the value of surface EPR becomes more with lower radius of the thin wire. However, after this period the value of surface EPR becomes less with lower radius of the thin wire.

It is shown from Figs. (8-10) that the effect of the radius of thin wires is clearer for arrangement " $\mathrm{A}$ " of the grounding electrodes and it is less clear for arrangement " $\mathrm{B}$ " and hybrid arrangement. This is attributed to the effect of horizontal 


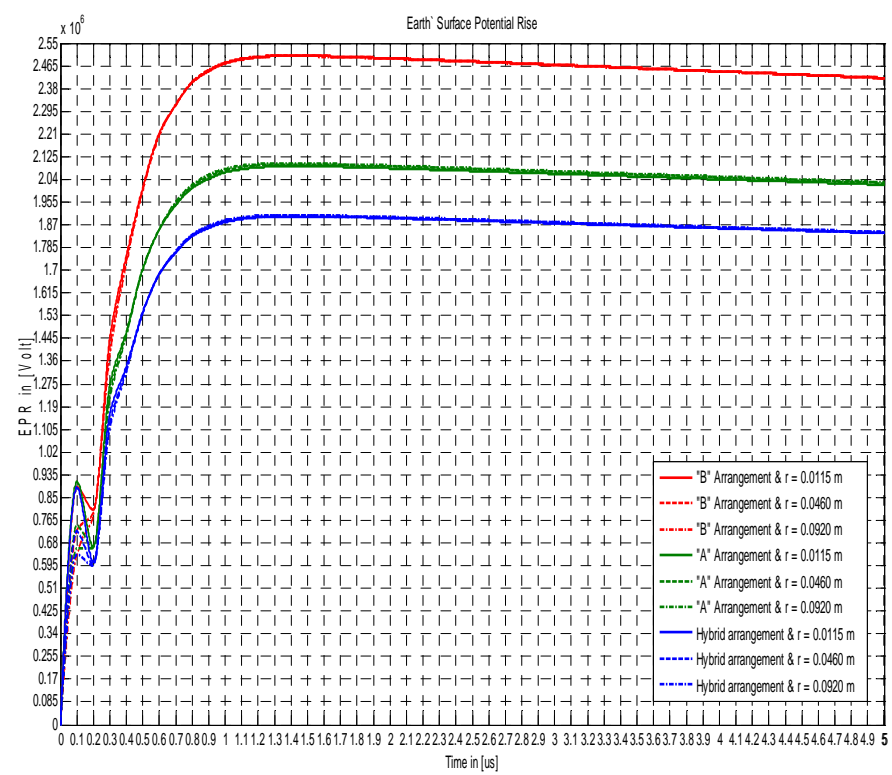

Fig. (8). The earth`surface potential rise with a soil resistivity of $2000 \Omega \cdot \mathrm{m}$.

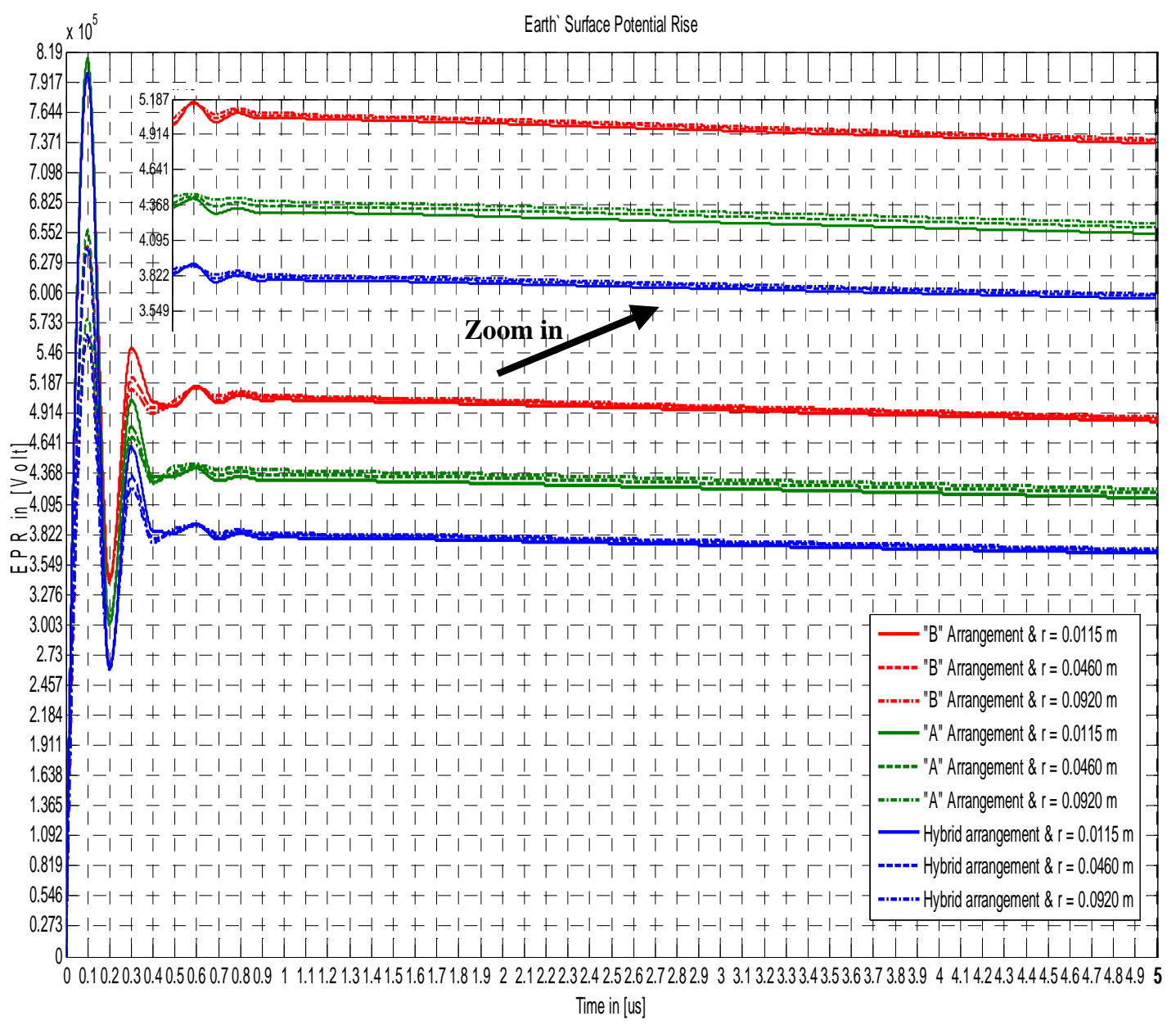

Fig. (9). The earth`s surface potential rise with a soil resistivity of $400 \Omega \cdot \mathrm{m}$.

equi-potential surface which is resulted from the horizontal copper grounding electrodes that is not existed in arrangement "A".

\subsection{Calculation of Transient Grounding Resistance}

In this section the transient grounding resistance (TGR) is calculated as explained in [15]. As mentioned in EPR 


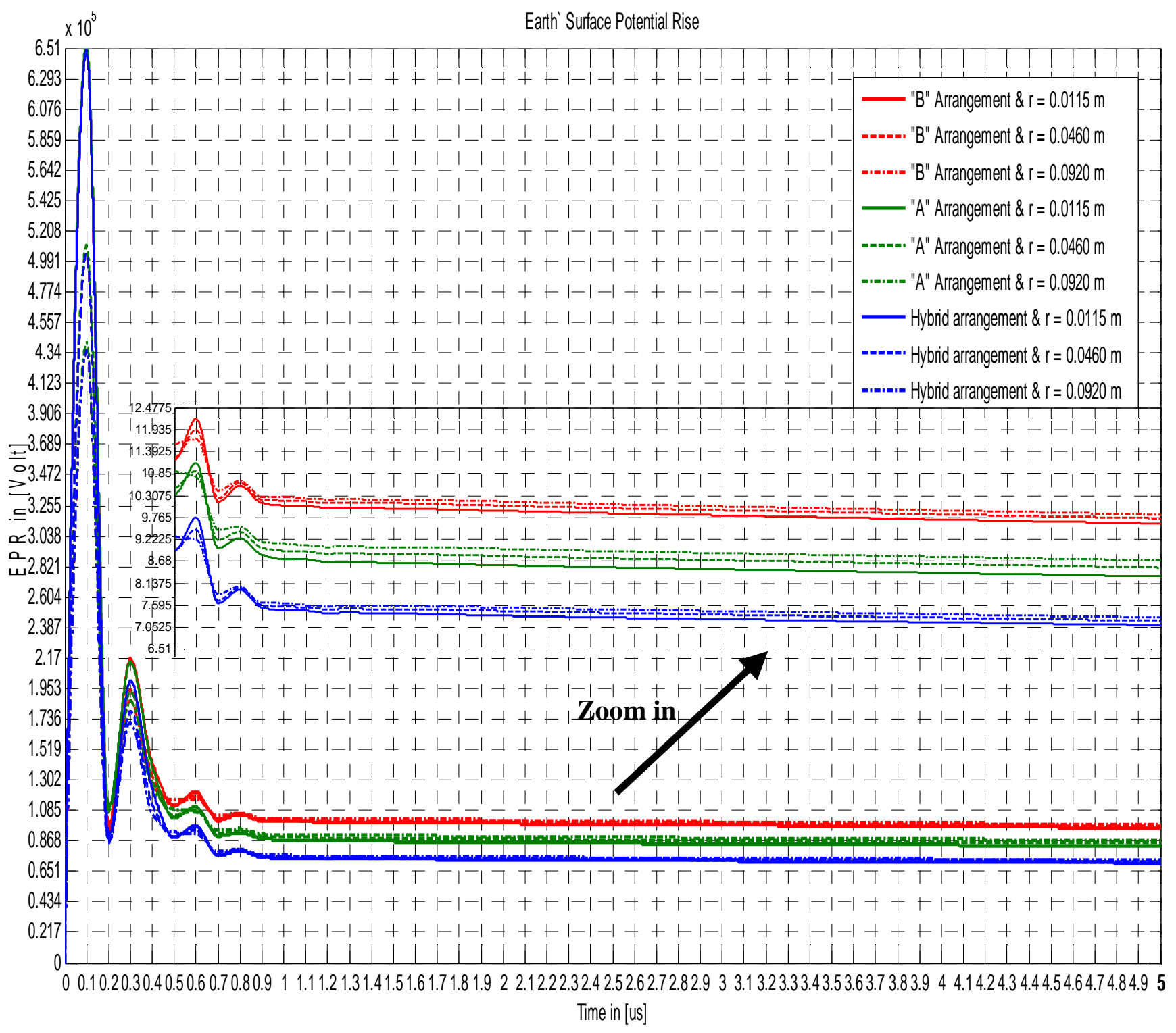

Fig. (10). The earth` surface potential rise with a soil resistivity of $80 \Omega \cdot \mathrm{m}$.

calculations, the TGR is calculated from a time instant of zero to $2.8 \mu \mathrm{s}$ for the three grounding arrangements which are shown in Fig. (4). For each one of these arrangements, the TGR is calculated at three values of the thin wire radius which are mentioned before in order to clarify the effect of changing the thin wire radius on TGR in FDTD calculations. Figs. (11-13) show the TGR at three values of soil resistivity which are $2000 \Omega \cdot \mathrm{m}, 400 \Omega \cdot \mathrm{m}$, and $80 \Omega \cdot \mathrm{m}$ respectively.

From these figures, it is shown that the steady state value of the grounding resistance occurs after about $1 \mu \mathrm{s}$ which is the front time of the lightning impulse current. A zoom in is done after the high value of the grounding resistance in both Figs. $(12,13)$ from the instant of $0.6 \mu$ s to $2.8 \mu \mathrm{s}$ in order to clarify the effect of changing the thin wire radius on the TGR.

From Figs. (11-13), the effect of the grounding electrodes arrangements on the grounding resistance is clarified as the hybrid arrangement gives the least value of the grounding resistance then arrangement " $A$ " and finally arrangement " $B$ ". The effect of changing the thin wire radius is clearer with less soil resistivity. It is shown that the value of the grounding resistance is more with less value of thin wire radius in the transient state up to about $0.5 \mu \mathrm{s}$. However, the value of the grounding resistance is less with less value of the thin wire radius. Furthermore, the effect of thin wire radius is clearer with arrangement " $\mathrm{A}$ " than that of both hybrid arrangement and arrangement " $\mathrm{B}$ ". 


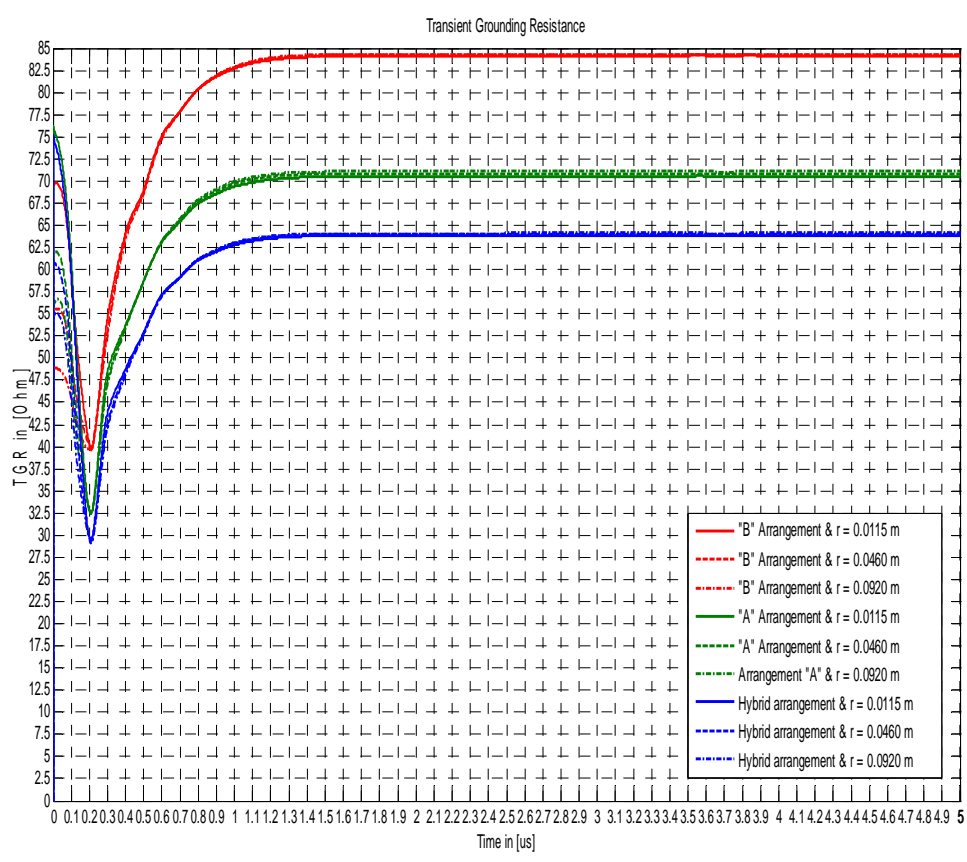

Fig. (11). The transient grounding resistance with a soil resistivity of $2000 \Omega \cdot \mathrm{m}$.

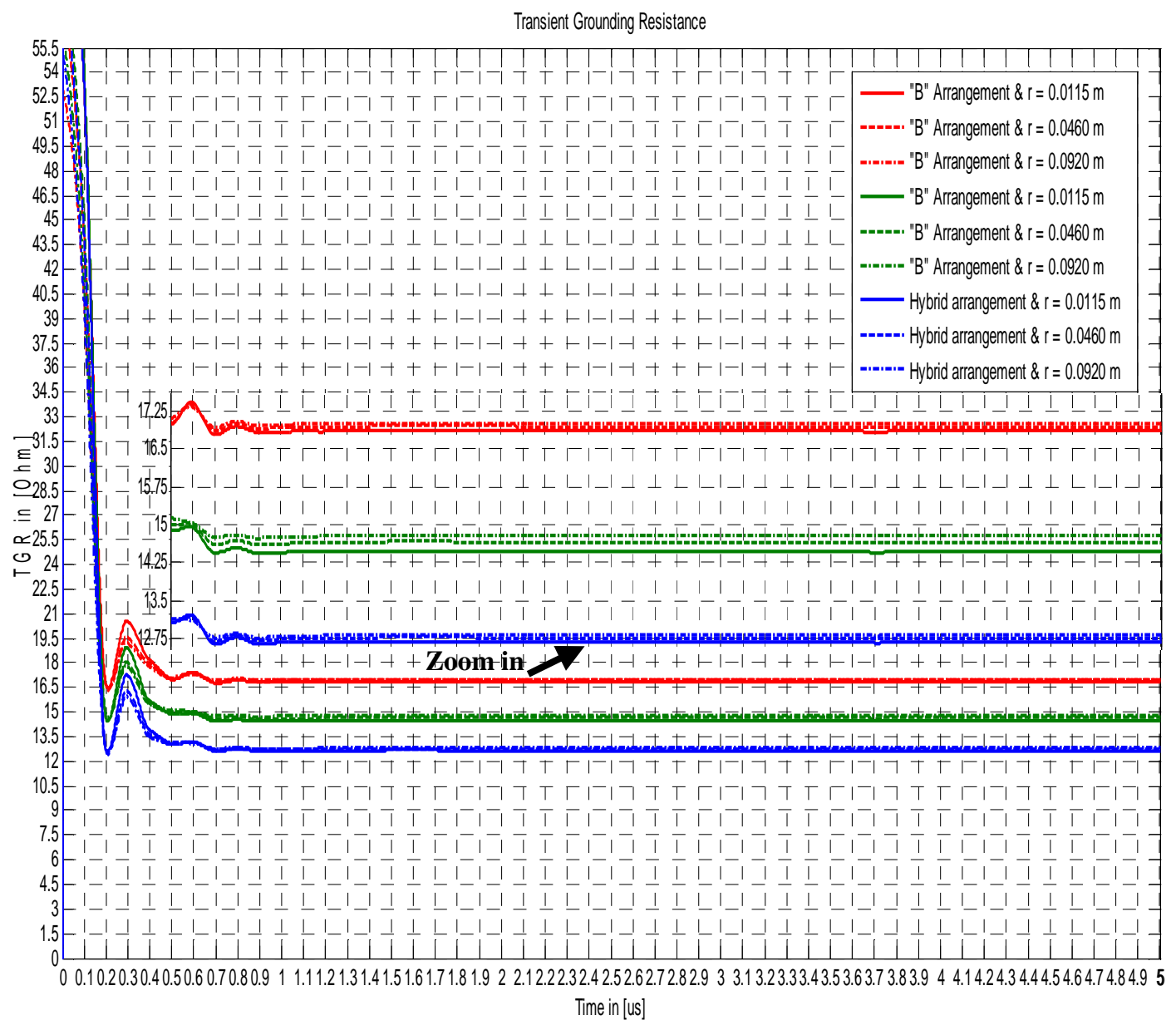

Fig. (12). The transient grounding resistance with a soil resistivity of $400 \Omega \cdot \mathrm{m}$. 


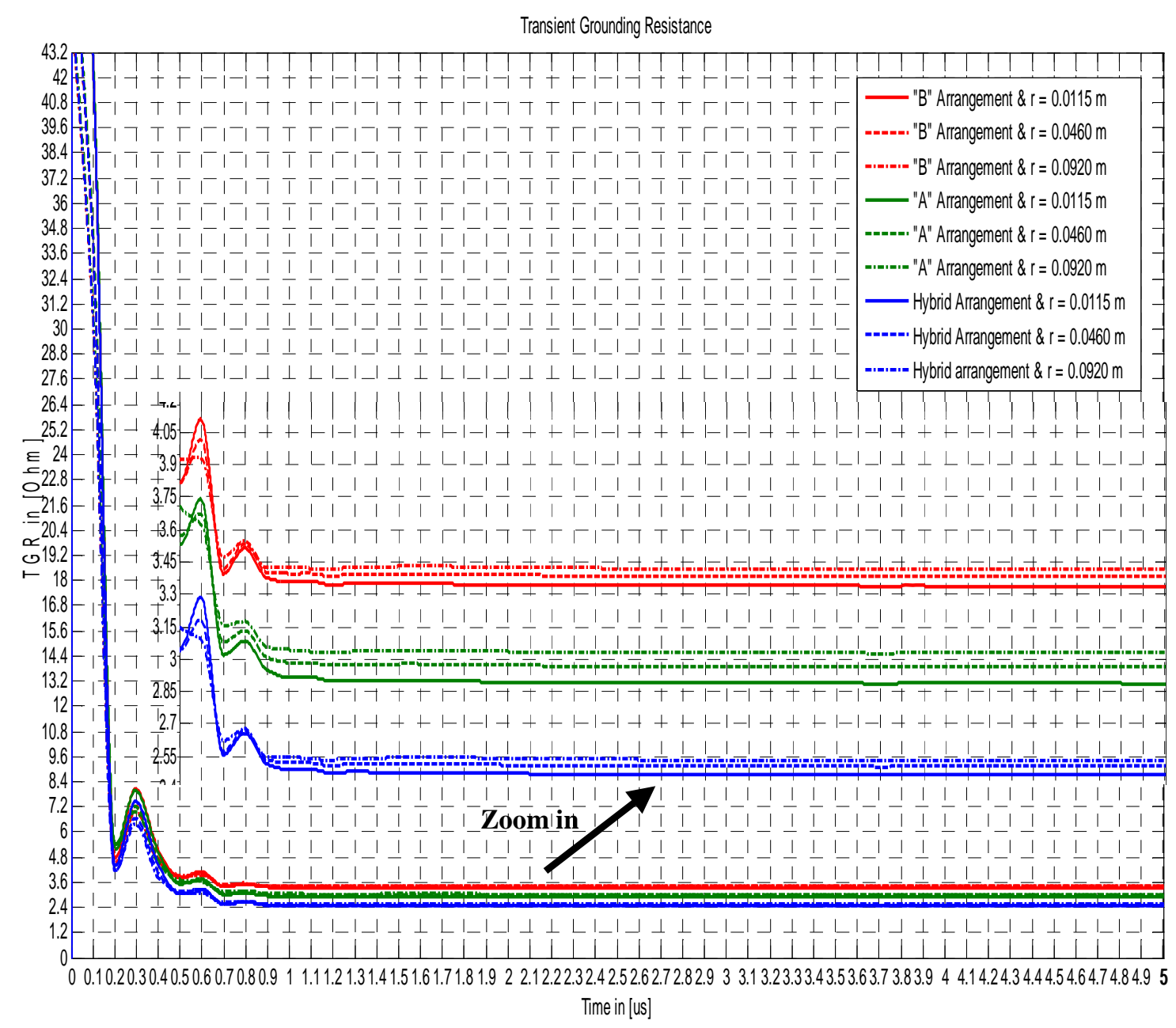

Fig. (13). The transient grounding resistance with a soil resistivity of $80 \Omega \cdot \mathrm{m}$.

\section{CONCLUSION}

In this paper, a detailed model is presented for IEC standards of earthing electrodes of the grounding systems of wind turbines. Finite difference time domain (FDTD) method is used to calculate electromagnetic fields due to direct lightning strikes on the grounding system. The electric field distribution in the grounding systems is shown using contour plots. Earth potential rise (EPR) and transient grounding resistance (TGR) are calculated from the calculated electromagnetic fields. The effect of radius of thin wires is demonstrated on electric field distribution, EPR, and TGR. The results show that the maximum value of the electric field in the grounding system is inversely proportional to the radius of thin wires. However; the rate of decrease in electric field with moving away from conducting points in the grounding system is less with higher thin wire radius. The results show that the value of both surface EPR and TGR in during transient state is more with less thin wire radius unlike its value during steady state. The impact of thin wire radius on both surface EPR and TGR is clearer at vertical earthing electrodes (Arrangement "A") than at horizontal earthing electrode (Arrangement "B") and horizontal earthing electrode with vertical earthing electrode (hybrid arrangement) due to the equi-potential surface caused by the horizontal earthing electrode.

\section{CONFLICT OF INTEREST}

The authors confirm that this article content has no conflict of interest.

\section{ACKNOWLEDGEMENTS}

Declared none.

\section{REFERENCES}

[1] GWEC. Global Wind Statistics (2012) [Online]. Global Wind Energy Council, Retrieved 2013, Nov 24 from website: http://www.gwec.net/wp-content/uploads/2013/02/GWEC-PRstats2012_english.pdf

[2] Yasuda Y, Uno N, Kobayashi H, Funabashi T. Surge Analysis on Wind Farm When Winter Lightning Strikes. IEEE Trans Energy Convers 2008; 23(1): 257262.

[3] Yasuda Y, Fujii T. Electromagnetic Calculation of a Wind Turbine Earthing System, Wind Turbines, Dr. Ibrahim Al-Bahadly (Ed.), ISBN: 978-953-307-221-0. InTech 2011; pp. 507-29.

[4] Yasuda Y, Fuji T, Ueda T. Transient Analysis of Ring Earth Electrode for Wind Turbine. In: Proceedings of European Wind Energy Conference, (EWEC'07), Milan, Italy 2007. 
[5] Yee KS. Numerical solution of initial boundary value problems involving Maxwell's equations in isotropic media. IEEE Trans Antennas Propag 1966; 14(3): 302-7.

[6] Noda T, Shigeru Y. Development of surge simulation code based on finite-difference time-domain (FDTD) approximation of Maxwell's equations. In: Proceedings of International Conference on Power System Transients, 2001 (IPST'01), Rio de Janeiro, Brazil 2001.

[7] Cigre Working Group C4.501. Guide for numerical electromagnetic analysis methods: application to surge phenomena and comparison with circuit theory-based approach 2013.

[8] Kuffel E, Zaengl W.S. High voltage engineering fundamentals. Pergamon Press 1984.

[9] Steven C, Chapra, Raymond PC. Numerical methods for engineers. $5^{\text {th }}$ ed. McGraw Hill Press 2006.

[10] Noda T, Yokoyama S. Thin wire representation in finite difference time domain surge simulation. IEEE Trans Power Delivery 2002; 17(3): pp. 840-7.

[11] Umran SI, Robert AM. Numerical electromagnetics. The FDTD Method. Cambridge University Press 2011.
[12] Karl SK, Raymond JL. The finite difference time domain for electromagnetics. CRC Press 1993.

[13] Stephen DG. Introduction to the finite-difference time-domain (FDTD) method for Electromagnetics. Morgan \& Claypool Press 2011

[14] Baba Y, Nagaoka N, Ametani A. Modeling of thin wires in a lossy medium for FDTD simulations. IEEE Trans Electromagnet Compatibility 2005; 47(1): 54-60.

[15] Run X, Bin C, Yun-Fei M, Wei D, Qun W, Yan-Yu Q. FDTD modeling of the earthing conductor in the transient grounding resistance analysis. IEEE Antennas Wireless Propag Lett 2012; 11 957-60.

[16] IEC. Protection against lightning-Part 3: Physical damage to structures and life hazard. International Electro-technical Commission. IEC 62305-3, Ed. 1.0(b). Geneva 2006.

[17] IEC. Wind Turbine Generation System-24: Lightning Protection. International Electro-technical Commission. IEC61400-24. Geneva 2010 .

(C) Rizk et al.; Licensee Bentham Open.

This is an open access article licensed under the terms of the Creative Commons Attribution Non-Commercial License (http://creativecommons.org/licenses/by-nc/3.0/) which permits unrestricted, non-commercial use, distribution and reproduction in any medium, provided the work is properly cited. 